\title{
Effect of Ambient Pressure Fluctuations on the Dual-Bell Transition Behavior
}

\author{
S.B.Verma ${ }^{\dagger}$ \\ Council of Scientific and Industrial Research (CSIR), National Aerospace Laboratories, \\ Experimental Aerodynamics Division, Bangalore-560017, India \\ R. Stark ${ }^{\star}$ and O. Haidn ${ }^{\S}$ \\ Institute of Space Propulsion, DLR Lampoldshausen, Germany - 74219
}

\begin{abstract}
An experimental investigation was conducted to study the effect of ambient pressure fluctuations on the dual-bell transition behavior. Ambient or back pressure fluctuations of different frequencies (1.0, 1.5 , and $3 \mathrm{~Hz}$ ) were artificially introduced inside the high-altitude simulation chamber using three magnetic valves. The dual-bell transition behavior is found to be very sensitive to back pressure fluctuations of magnitude $>20 \%$ which triggers a flip-flop phenomenon that is observed to continue as long as the fluctuations prevail. Increasing the throttling up rate also does not seem to help much in achieving a stable dual-bell transition within the scope of the present tests. This too is not favorable as it can lead to a delay in transition. These test results suggest that the dual-bell nozzle can present problems in real-flight operation especially when the launcher experiences the buffeting phase of flight. The solution perhaps lies in increasing the dual bell stability parameter to a value greater than the magnitude of back pressure fluctuations so as to avoid the nozzle pressure ratio range that will set the dual-bell into a non-stop flip-flop mode during buffeting.
\end{abstract}

\section{Nomenclature}

$\alpha_{i} \quad=$ wall angle at the inflection point, degrees

$\alpha_{e} \quad=$ wall angle at nozzle exit, degrees

$\epsilon \quad=$ area-ratio of the dual-bell nozzle

$\epsilon_{b} \quad=$ area-ratio of the base nozzle

$f \quad=$ frequency of back pressure fluctuations, $\mathrm{Hz}$

$\dot{m} \quad=$ mass flow rate of test gas, $\mathrm{kg} / \mathrm{s}$

$P_{a} \quad=$ ambient pressure for tests in sea-level atmospheric conditions, bar

$P_{b} \quad=$ back pressure measured inside the high-altitude test chamber, bar, Fig. 2 (a)

$P_{O N} \quad=$ stagnation pressure of the dual-bell test nozzle, bar

\footnotetext{
† Principal scientist, CSIR-NAL Bangalore, Associate Fellow AIAA, E-Mail: sbverma@nal.res.in

\$Head, Nozzle Flow Group, DLR Lampoldshausen, E-Mail: ralf.stark@dlr.de

$\S$ Professor, Technical University München, Fellow AIAA, E-Mail: oskar.haidn@dlr.de
} 
$P_{O E} \quad=$ stagnation pressure of the ejector nozzle, bar

$P_{w} \quad=$ local wall pressure, bar

$\left|\partial P_{O N} / \partial t\right|=$ rate of change in dual-bell nozzle feeding pressure, bar/s

$r_{t} \quad=$ radius of the nozzle throat, $\mathrm{mm}$

$t \quad=$ time at a particular sequence of event, $\mathrm{s}$

$X \quad=$ co-ordinate along the nozzle axis, $\mathrm{mm}$

$\mathrm{DB}_{\mathrm{st}}=$ dual-bell stability parameter

NPR = nozzle pressure ratio, $P_{0 N} / P_{a}$ or $P_{0 N} / P_{b}$

$\mathrm{NPR}_{\mathrm{tr}}=$ forward transition NPR of dual-bell nozzle

$\mathrm{NPR}_{\mathrm{re}-\mathrm{tr}}=$ backward or re-transition NPR of dual-bell nozzle

TIC $=$ truncated ideal contour

HASC $=$ high altitude simulation chamber

\section{Introduction}

The key requirement for the main engines of heavy launch vehicles such as the Space Shuttle and Ariane 5 is a high vacuum performance as they have to operate from sea-level to near vacuum conditions. The maximum geometric area-ratio available for such engines is constrained by flow separation and the associated side-loads during transient start up at sea-level ignition and for upper stage applications by the available integration volume or the storage space. Other than the geometric constraints, the bell nozzles owing to their fixed geometry also limit the overall engine performance during the launcher's ascent.The challenge of improving the vacuum performance of these main engines has led to the concept of altitude compensating nozzles aimed at closing the gap between the present and ideal engines. Various concepts have been discussed in the past and tested on ground. These include the extendible nozzle [1] (such as RL-10B-2 and RL10A-4-2 being widely used for upper stage applications), dual-bell nozzle [2-3] with single-step altitude adaptation capability, annular/linear-plug nozzle [4-7] for first or upper-stage application, Znamensky or `Z-nozzle’ (zeroplug length) [8], and expansion-deflection thrust chamber [5,9] for upper stage which offers substantial decrease in integration volume without any moving parts. Other than the extendible nozzle, none of these concepts have ever reached flight hardware status. On the other hand, the dualbell nozzle, due to its simple design and being similar to the conventional bell-nozzle, requires minimum changes on the existing launcher configurations making it the most feasible altitude adaptive nozzle in the present conditions.

The concept of dual-bell nozzle, first proposed by Foster and Cowles in 1949 [2] has received renewed attention in recent years due to its one-step altitude adaptation capability [2-3, 10-13].This concept uses two shortened nozzles combined into one with a bump or inflection point between them, 
as shown in Fig. 1. During ascent it functions first at the lower area-ratio with controlled flow separation occurring at the inflection point, Fig. 1 (a). While the lower area-ratio helps to achieve high sea-level thrust, a controlled and symmetrical flow separation helps avoid dangerous side-loads. As the altitude increases and the gases expand further, the flow undergoes a transition process during which the flow jumps downstream and attaches itself close to the nozzle exit, with the flow filling the full nozzle exit section thereby utilizing the full geometrical area-ratio, Fig. 1 (b). Because of the higher area-ratios that are achievable through this design, a higher vacuum performance is feasible. Despite the losses associated with this design (such as due to aspiration drag in low-altitude mode, non-optimum contour in high altitude mode, etc. [13]), the dual-bell nozzle still shows better overall performance than a single bell nozzle of similar area-ratio [3].

Recent studies at DLR Lampoldshausen have indicated that as the nozzle pressure ratio (NPR) of the dual-bell approaches the transition value, the nozzle first approaches the phase of 'sneak' transition [14-15] wherein the separation point begins to gradually move from the inflection point to the first point in the nozzle extension (i.e., in the region of negative pressure gradient, time duration $t_{t r, l}$ ) after which it finally accelerates in the nozzle extension in a very short time interval (time duration $t_{t r, 2}$ ). The transition of the separation point in the inflection region, which is characterized by a negative pressure gradient, like in conventional nozzles, has been found for the first time in a numerical study reported in ref. [16] which was later confirmed experimentally in refs. [14-15]. The former transition time $t_{t r, l}$ is found to be sensitive to the wall inflection design, local Reynolds number of operation and secondary-film cooling which modifies the width of the region of wall inflection [16,19-20]. The latter transition time $\left(t_{t r, 2}\right)$, on the other hand, has been reported [17] as the most important parameter characterizing the transition behavior since the separation front during this time moves a significant portion of the nozzle extension. It has been observed that increasing the rate of ramp-up gradient $\left(\partial P_{0} / \partial t\right)$ reduces this transition time which is preferred from the viewpoint of reducing side-loads during the transition modes [17]. In real flight, a fast transition can be achieved by lowering the chamber pressure initially and then increasing it fast enough so as to ensure an abrupt change in NPR [18]. In addition to the above studies, recent studies at DLR Lampoldshausen have also focused on improving the stability of the dual-bell transition [14-15]. The stability here implies in increasing the gap between the transition and re-transition NPR so as to prevent oscillations from one mode to the other that might be induced either by variations in $P_{0}( \pm 5 \%)$ due to combustion instabilities or by $P_{a}$ variation due to buffeting effect in real-flight. These studies have indicated that increasing the relative length of the nozzle extension (as compared to other test nozzle models which have similar base nozzle length but different nozzle extension lengths) helps improve the transition stability of the dualbell nozzle. Control of transition NPR has also been demonstrated using secondary injection at the wall inflection location with the secondary mass flow ratio as the controlling parameter [19]. 
However, most of the previously reported results are from tests conducted in the absence of ambient pressure fluctuations until recently [21-22]. Under real-flight conditions, however, the launch vehicle experiences significant ambient pressure fluctuations while passing through the buffeting phase of its flight. The Ariane 5 experience [23] shows that the vehicle base region experiences an $r m s$ fluctuation of $60 \mathrm{mbar}(f \sim 100 \mathrm{~Hz})$ at $9 \mathrm{~km}$ altitude where the ambient pressure $\left(P_{a}\right)$ is of the order of $300 \mathrm{mbar}$. This shows $20 \%$ fluctuations in the value of $P_{a}$. Since the dual-bell transition is sensitive to changes in NPR, it becomes mandatory to study the dual-bell transition behavior in the presence of such $P_{a}$ fluctuations. Ambient or back pressure $\left(P_{b}\right)$ fluctuations of different frequencies $(f=0.5,1.0,1.5,2.0$ and $3 \mathrm{~Hz}$ ) were artificially introduced in the high-altitude simulation chamber (HASC) using a combination of 3 magnetic valves. Such a test campaign has been attempted for the first time inside a HASC to study the dual-bell transition stability behavior simulating real-flight conditions. The primary objective was to study the effect of varying the (i) frequency $(f)$ of back pressure fluctuations, (ii) magnitude of back pressure $\left(\Delta P_{b}\right)$ fluctuations and, (ii) throttling up rate or the rate of change in $P_{O N},\left|\partial P_{O N} / \partial t\right|$, on the dual-bell transition behavior. Within the experimental limitations, it was found that for $P_{b}$ fluctuation magnitudes greater than $20 \%$ the dual-bell is set into a flip-flop phenomenon which continues as long as the $P_{a}$ fluctuations exist.

\section{Experimental Set Up and Procedure}

\section{A. Test Facility}

Tests were carried out in the subscale cold flow test facility P6.2 in DLR Lampoldshausen inside a high-altitude simulation chamber (HASC), as shown in Fig. 2 (a). The altitude chamber was evacuated using an additional ejector nozzle. Gaseous nitrogen at ambient temperature is used as the test gas due to its advantage over compressed air. This is primarily due to the lower saturation temperatures associated with pure nitrogen. The throat diameter of the sub-scale dual-bell nozzle used for the experimental investigation was $20 \mathrm{~mm}$, yielding maximum mass flows in the range of $\mathrm{m}=4.2 \mathrm{~kg} / \mathrm{s}$ under facility operating limitations. Figure 2 (b) shows the location of pressure transducers on the test model and Table 1 shows the important geometric nozzle parameters, respectively. The contour of the dual-bell base nozzle was designed as a truncated ideal contour (TIC) while that of the nozzle extension was designed on the isobar leaving the last point of the base nozzle using method of characteristics featuring a constant pressure profile (CP) [4]. The ejector nozzle used is a thrust optimized parabolic nozzle $\left(M_{d}=3.2\right.$, NPR for full-expansion 49.5$)$ and supplied with an additional nitrogen feed line with an independent pressure regulating valve.

Back pressure fluctuations of different frequencies $(f=0.5,1.0,1.5,2.0$ and $3 \mathrm{~Hz})$ were artificially introduced in the high-altitude simulation chamber (HASC) using magnetic valves, as shown in Fig. 3 (a). Three such COAX co-axial externally controlled valves (specification VMK 25 DR) were 
installed on the top wall of the HASC, Fig. 3 (b). The $P_{b}$ in the altitude chamber was measured only at one point which is on the top wall of the chamber and not at multiple points. However, it may be emphasized that the wall pressure is measured near the DB nozzle exit at $X / r_{t}=13.2$ (at 4 locations around the nozzle circumference with one Kulite transducer placed in each quadrant, e.g, locations 13 and 13Q2 as seen in Fig. 2 (b)) during each run. Each of these transducers show very similar value which is almost $96 \%-97 \%$ of $P_{b}$ (in low-altitude mode) measured on the top wall of the chamber. This clearly indicates a uniform distribution of $P_{b}$ inside the HASC during the tests. In the present set up, the valves were kept open to the atmosphere and no additional air supply was supplied to the valves. Initially it was assumed that the combination of all three valves would give enough mass flow supply into the HASC that would be sufficient to effect significant changes in $P_{b}$ to induce dual-bell transition. However, when these valves are operated at $f \geq 3 \mathrm{~Hz}$, it was observed that the changes in back pressure $\left(\Delta P_{b}\right)$ induced inside the HASC (in subsequent fluctuations) were not of sufficient magnitude to initiate a dual-bell transition. As a result, tests for $f>3 \mathrm{~Hz}$ were abandoned. Tests were also conducted for various throttle-up and throttle-down rates, $\left|\partial P_{0} / \partial t\right|$ (of $0.5 \mathrm{bar} / \mathrm{s}, 1 \mathrm{bar} / \mathrm{s}$ and $2 \mathrm{bar} / \mathrm{s}$, respectively) in the presence of $P_{b}$ fluctuations. It may however be emphasized here that although higher $P_{b}$ fluctuation frequency could not be achieved under the limitations of the present test set up, the major concern here was more on simulating the amplitude of pressure fluctuations (of 20\%). This is because the DB nozzle is sensitive to changes in NPR due to its inherent design. On the other hand, higher fluctuation frequencies with sufficient amplitude would further add to the seriousness of the situation.

\section{B. Data Acquisition System}

The data acquisition system used for the pressure measurements has the capacity of measuring 64 channels at $1 \mathrm{kHz}$ and 16 at $50 \mathrm{kHz}$ or 8 at $100 \mathrm{kHz}$. A low-pass filter cut-off frequency of $160 \mathrm{~Hz}$ is used for $1 \mathrm{kHz}$ acquisition and of $8 \mathrm{kHz}$ for high frequency acquisition, respectively. For real-time wall pressure measurements fast piezo-resistive pressure sensors 'Kulite Semi-conductor Inc.' transducers are used (model XT-154-190M). Three such pressure sensors are placed in the in the base nozzle while four are located in the vicinity of the nozzle wall inflection in each of the four quadrants to monitor the movement of separation front during transition, Fig. 2 (a). Ten sensors are also placed along a single axial line in the nozzle extension. These transducers, with a pressure-sensitive area of $0.4 \mathrm{~mm}^{2}$ and an outer case area of $12 \mathrm{~mm}^{2}$, have a natural frequency of $50 \mathrm{kHz}$. The accuracy is within $0.5 \%$ in the operating pressure range of $0.1 \mathrm{MPa}$, with a sensitivity of typically $0.97 \mathrm{~V} / \mathrm{MPa}$. The transducers are threaded into the nozzle wall. A small orifice (of $1 \mathrm{~mm}$ length and $0.5 \mathrm{~mm}$ diameter) connects the transducer to the flow. This configuration results in an estimated resonance frequency of $68.75 \mathrm{kHz}$. The wall pressure data for all the pressure transducer locations was acquired at $20 \mathrm{kHz}$. 


\section{Results and Discussions}

\section{A. Streamwise Distribution of Mean Pressure}

Figure 4 shows the streamwise distribution of mean wall pressure in a dual-bell nozzle for three operating conditions, namely (i) with separation point at the inflection point, (ii) with separation point inside the region of wall inflection and, (iii) under fully attached flow condition post-transition. These three conditions correspond to the low-altitude mode, "sneak" transition [14-15,21] (a condition prevalent just before transition during which the separation front moves into the region of wall inflection but is not yet pushed into the nozzle extension [17]), and the high-altitude mode after transition, respectively. These results are from tests conducted under sea-level conditions [17]. During low-altitude operation, a controlled separation is seen to occur at the wall inflection location that is followed by a gradual pressure adaptation to the ambient pressure occurring over the remaining length of the nozzle extension. As the NPR is increased and the overexpansion levels for the base nozzle decrease, the separation point gradually moves into the region of wall inflection at NPR $=49.1$ (at $X / r_{t}=6.11$ and marked by a vertical dashed line), i.e., just before transition occurs. Sneak transition here is defined as the flow condition wherein the incipient separation location begins to gradually move or 'sneak' into the region of wall inflection before it finally jumps downstream [14-15, 21]. This condition is identified [21] by a lower pressure $\left(P_{w}\right)$ adaptation in the back-flow region to $P_{a}$ or $P_{b}$ and is caused due to reduction in the lateral extent of the back-flow or re-circulation zone [17]. And finally during the process of forward transition ( $\mathrm{NPR}=49.2)$, the separation point jumps downstream from the wall inflection location to the nozzle exit after which the nozzle extension shows a constant wall pressure distribution as seen for NPR $=50.5$.

It may be pointed out that during the process of sneak transition, the separation front moves gradually downstream asymmetrically around the wall inflection location before it finally experiences the downstream jump at the design NPR. The trend in which the separation front moves asymmetrically downstream is similar in all test runs for this test article. This is observed both without and with $P_{b}$ fluctuations. However, it may also be pointed out that such a trend has also been observed during sealevel tests where the same test article is not placed vertically inside the HASC but on a horizontal test bed [14-15, 17]. Also as pointed out earlier, the $P_{b}$ distribution inside the HASC and around the nozzle exit is nearly uniform. So the asymmetric movement of the separation front with or without the $P_{b}$ fluctuations seems more due to the inherent nature of shock front that is asymmetric before any transition process whether it is in the case of a DB nozzle [14-15, 17] or a single bell nozzle such as that reported earlier in a thrust optimized parabolic [24] or in a truncated ideal contour nozzle [25].

During re-transition, the reverse phenomenon occurs, i.e., the dual-bell undergoes a transition from the high-altitude mode to low-altitude mode. The re-transition however occurs at a much lower NPR 
of about 39.2 [17]. It can be seen that the wall pressure distribution undergoes significant changes as the dual-bell undergoes transition from the low-altitude mode to high-altitude mode and vice-versa. As a result, both during transition and re-transition, the dual-bell experiences side-loads [14-18] that are unavoidable.

To study the effect of ambient pressure fluctuations on the dual-bell transition behavior, the tests were however conducted inside a HASC where the fluctuations in $P_{b}$ could easily be introduced.

\section{B. High Altitude Simulation Chamber}

Usually a high altitude test facility comprises of three major-components; (i) a closed chamber in which low pressure environment is desired, (ii) supersonic ejector nozzle or test-nozzle, and (iii) supersonic exhaust diffuser, Fig. 2 (a). As the ejector/test nozzle is fed with driving pressure, the supersonic exhaust from the nozzle exit begins to evacuate the closed chamber by entraining the air that is driven by (i) the difference in pressure between the nozzle exit and the altitude chamber, and (ii) momentum transfer by the high-speed nozzle exhaust [7-8]. This initiates a fluid motion in the direction of pressure and momentum gradients established in the test set up. The initial suction during the starting transient evacuates the altitude chamber to low pressures that provides high-altitude environment for nozzle operation, Fig. 5 (a). The diffuser operation is broadly characterized by two distinct operating modes, i.e., diffuser unstart-mode and diffuser start-modes [8]. Figure 5 (b) shows an illustration of a typical chamber pressure history during diffuser operation. In the diffuser un-start mode, the ejector driving pressure $\left(P_{0}\right)$ is not high enough to initiate diffuser starting condition and the nozzle operates in over-expanded condition with the jet exhaust characterized by a Mach disk (mode A). In an intermediate operating condition, the test nozzle reaches full-expansion and a transition from Mach reflection to regular reflection occurs (mode B) but the diffuser is still in un-start condition. And finally, in diffuser-starting mode the ejector nozzle operates in highly under-expanded condition with the jet boundaries impinging on the diffuser inlet wall (mode $\mathrm{C}$ ). In this condition the altitude chamber gets isolated from the ambient. Once this happens the evacuation process in the altitude chamber is no longer affected by the ambient pressure changes and thus a sudden drop in $P_{b}$ occurs, Fig. 5 (b). Such an arrangement provides moderate evacuation levels while mimicking low pressure environment at high altitudes.

Figure 6 (a) shows the pressure time-history of one of the runs made with the present test set up. Both the ejector and dual-bell nozzle are started simultaneously. After reaching a certain value, the dual bell $P_{O N}$ is held constant while the ejector $P_{O E}$ continues to increase up to $30 \mathrm{bar}$. Once ejector $P_{O E}$ is held constant and the HASC is evacuated to a certain $P_{b}$ value, the dual-bell feeding pressure $P_{O N}$ is throttled up and down to achieve transition, Fig. 6 (a). Figure 6 (b) shows the change in $P_{b}$ values introduced in the HASC as the magnetic valves (MVs) are switched on one after the other in the 
absence of dual-bell operation. Significant increase in $P_{b}$ values can be seen with the switching on of each MV. However in real-flight conditions, $P_{O N}$ remains constant while the ambient pressure or $P_{b}$ decreases as the launcher ascents in the atmosphere. Therefore for subsequent tests, as shown in Fig. 7 (a), $P_{O N}$ was held constant while $P_{b}$ pressure fluctuations were introduced that simulates real-flight situation experienced during the buffeting phase of flight. However, with dual-bell operation, the effective $P_{b}$ value that is attained before the MVs are switched on is much higher due to the additional mass flow (from test nozzle) being continuously added inside the HASC. As a result, when all three MVs are switched on (with dual-bell operating), a similar initial $P_{b}$ value could not be attained as observed before (76mbar was achieved as opposed to 12.6mbar), as can be seen in Figs. 6 (b) \& 7 (b).

\section{Effect of Back Pressure Fluctuations}

Figure 7 (a) shows the fluctuations in $P_{b}$ obtained in response to the pressure fluctuations introduced with all the three MVs in operation. Frequencies of $1 \mathrm{~Hz}, 1.5 \mathrm{~Hz}$ and $3 \mathrm{~Hz}$ are introduced independently during the same run. It can be seen that the dual-bell transition is very sensitive to fluctuations in $P_{b}$ value. For each of these frequencies, although the initial increase in $P_{b}$ value is similar (from $76 \mathrm{mbar}$ to $104.3 \mathrm{mbar}$ ), successive frequencies of $P_{b}$ fluctuations are seen to result in a change in $P_{b}$ value of 24.3mbar (30\%), 20.3mbar (24\%) and 7.3mbar (7\%), respectively, Fig. 7 (b). Each time a pressure fluctuation is introduced in the HASC, the dual-bell is seen to undergo a full transition followed by a re-transition. For frequencies below $2 \mathrm{~Hz}$, the dual-bell is seen to go into a non-stop flip-flop mode wherein the nozzle continuously fluctuates between the low- and high-altitude modes as long as the pressure fluctuations prevail inside the HASC. It can also be seen that when the percentage fluctuation in $P_{b}>20 \%$ (which is the magnitude of $P_{a}$ fluctuations experienced in the Ariane 5 base region) the dual-bell operation is susceptible to a highly unstable flow condition. This flow condition is not desirable for the successful operation of dual-bell nozzle especially when the launch vehicle passes through the buffeting phase of its flight regime as this can significantly affect the nozzle performance and can lead to nozzle structural failure. However, for $f=3 \mathrm{~Hz}$, the magnitude of pressure fluctuations (7\%) introduced were not sufficient enough to trigger a full transition although the $P_{w}$ pressure in the nozzle extension continued to fluctuate at the same frequency. Although switching on the MVs initially increased the $P_{b}$ value as expected (to $104.3 \mathrm{mbar}$ for $3 \mathrm{~Hz}$ ), the time interval between subsequent switching on and off operation (due to the relatively higher frequency) however does not give sufficient time to the ejector to evacuate the HASC to a much lower pressure before encountering the next fluctuation. As a result pressure fluctuations of the order of only $7 \%$ could be achieved and so no full transition could be seen for frequencies higher than $1.5 \mathrm{~Hz}$.

In order to investigate the importance of the magnitude of the back pressure fluctuations towards initiating an unstable DB condition, an additional test was conducted, as shown in Fig. 7 (c). In this 
test, only two MVs were operated as opposed to three MVs used earlier while keeping the test sequence and frequency operation similar, as seen in Fig. 7 (a). The $P_{b}$ value achieved with the MVs switched off is similar (76mbar) as in previous test while its value after switching on the $2 \mathrm{MVs}$ is 99.5mbar. The latter value is lesser as expected than the value achieved when $3 \mathrm{MVs}$ were used, Fig. 7(b). As a result, the use of $2 \mathrm{MVs}$ affects the amplitude of subsequent fluctuations for similar frequencies. It can be seen in Fig. 7 (c) that the magnitude of $P_{b}$ variations achieved for $1 \mathrm{~Hz}$ sequence is only $21 \%$ while that for $1.5 \mathrm{~Hz}$ and $3 \mathrm{~Hz}$ as $16 \%$ and $4 \%$, respectively. These values are much lower than when $3 \mathrm{MVs}$ were used, Fig. 7 (b). It can be seen that only the $1 \mathrm{~Hz}$ frequency operation is able to initiate the flip-flop behaviour. For relatively high frequencies of $1.5 \mathrm{~Hz}$ and $3 \mathrm{~Hz}$, the magnitude of $P_{b}$ variation achieved with $2 \mathrm{MVs}$ operating is much lesser and is not sufficient to create an unstable flow condition observed earlier. This is in contrast to the observation with $3 \mathrm{MVs}$ where the $1.5 \mathrm{~Hz}$ operation also shows a successful flip-flop behavior. The results of Figs. 7 (b) and (c) clearly indicate that the magnitude of $P_{b}$ variations is an important parameter in addition to its frequency and cannot be ignored.

It may be noted that in the case of a full-scale nozzle, since the length of the second bell would be much longer, the total transition time would also be longer relative to the present subscale nozzle (assuming transition velocity to be constant for a subscale and full-scale nozzle). Therefore the critical frequency, based on the time duration of forward transition and re-transition, would also be lower. However, more tests in the future need to be conducted to corroborate the above and is beyond the scope of the present work.

\section{Effect of Throttling Up}

Tests were also conducted to investigate the effect of throttling $P_{O N}(0.5 \mathrm{bar} / \mathrm{s}$ and $2 \mathrm{bar} / \mathrm{sec})$ in the presence of $P_{b}$ fluctuations on the dual-bell transition behavior, Fig. 8. For these tests, the fluctuations in $P_{b}$ were introduced 2-3 seconds before the process of throttling up was finally initiated. This simulates the real-flight scenario wherein the engine exhaust begins to experience the $P_{a}$ fluctuations during buffeting phase and the engine throttle control is initiated to help the dual-bell reach a successful and a stable transition. The present tests indicate that at lower frequencies of $1 \mathrm{~Hz}$, once the flip-flop behavior is initiated it continues to prevail even while throttling-up and throttling-down the test nozzle $P_{O N}$. However for higher frequencies $(>1.5 \mathrm{~Hz})$, once again, although significant fluctuations in $P_{w}$ in the nozzle extension can be felt, no full transition is achieved. This behavior too is not favorable as it can lead to a delay in dual-bell transition. Increasing the throttle rate (to $2 \mathrm{bar} / \mathrm{s}$ ) too does not seem to help achieve a full transition, Fig. 8 (b). But in either case the $P_{w}$ in the entire nozzle extension continues to fluctuate significantly and is not acceptable from the view point of sideloads. A careful observation once again shows that whenever the magnitude of $P_{b}$ fluctuations exceeds 
$20 \%$, the flip-flop behavior gets initiated irrespective of the rate of throttle up or throttle down sequence. These test results clearly indicate that the issue of $P_{b}$ fluctuations is important and that a lot of care needs to be taken while designing the dual-bell nozzle for real-flight situation so as to ensure a stable forward transition. The tests also suggest that for $f>2 \mathrm{~Hz}$ increasing the throttling up rate does not seem to help much in achieving dual-bell transition in the present test arrangement.

The above study clearly suggests that the dual-bell stability parameter defined $[14-15,17]$ as $\left[\mathrm{DB}_{\mathrm{st}}=\right.$ $\left(\left(\mathrm{NPR}_{-}\right.\right.$tr $-\mathrm{NPR}_{-}$retr $\left.\left.) / \mathrm{NPR}_{\text {tr }}\right)\right]$ is an important parameter that can prevent an unstable or a non-stop flip-flop type of situation in real-flight condition. For the present test nozzle, although the design $\mathrm{NPR}_{-\mathrm{tr}}=49$ and $\mathrm{NPR}_{-}$retr $=39$, the NPR_tr and NPR_retr achieved with the operating conditions in HASC is 55 and 44, respectively. This is primarily due to the Reynolds number effect that becomes predominant for tests inside the HASC, as reported earlier [26]. Despite the variations in the achieved values of $\mathrm{NPR}_{-}$tr and $\mathrm{NPR}_{-}$retr, the $\mathrm{DB}_{\text {st }}$ evaluated from these numbers suggests a value of approximately $20 \%$. In the present test campaign, it is observed that for $P_{b}$ fluctuations $>20 \%$, the dual-bell is set into a continuous flip-flop type of situation. Further the results in Figs. 7 (b) and (c) clearly indicate that the magnitude of $P_{b}$ variation is also an important parameter in addition to its frequency. This means that if such an unstable situation is to be avoided, then for a known value of $\%$ $P_{b}$ fluctuations, the $\mathrm{DB}_{\mathrm{st}}$ value should be much larger than the magnitude of these fluctuations. Since for the Ariane 5 launch vehicle, the flight data shows $20 \%$ ambient pressure fluctuations felt in the base region, the dual-bell therefore has to be designed for a $\mathrm{DB}_{\mathrm{st}}$ value greater than $20 \%$. So for a 300mbar $P_{a}$ buffeting situation, the preliminary estimations suggest that the dual-bell (operating with a chamber pressure $\left(P_{c}\right)$ of 117bar, same as Vulcain 2) has to be designed for an NPR $\mathrm{Nr}_{-}>440$ and an NPR ${ }_{\text {retr }}$ of 350 . Whether the throttling-up of $P_{c}$ before the beginning of buffeting or during buffeting will help avoid this adverse situation needs to be further thoroughly investigated. It can also be concluded that for dual-bell nozzle the magnitude of $P_{b}$ fluctuations is also an important parameter in addition to its frequency and cannot be ignored.

\section{Conclusions}

An experimental investigation has been conducted to study the dual-bell transition behavior in the presence of ambient pressure fluctuations simulating real-flight conditions. A combination of three magnetic valves were used to artificially create back pressure fluctuations (of $f=0.5,1.0,1.5,2$ and $3 \mathrm{~Hz})$ inside the HASC. The primary objective was to study the effect of varying the (i) frequency $(f)$ of back pressure fluctuations, (ii) magnitude of back pressure $\left(\Delta P_{b}\right)$ fluctuations and, (ii) throttling up rate or the rate of change in $P_{O N},\left|\partial P_{O N} / \partial t\right|$, on the dual-bell transition behavior. 
The present tests indicate that the dual-bell transition behavior is very sensitive to $P_{b}$ fluctuations. Each time the magnitude of back pressure pulsations $\Delta P_{b}$ exceed $20 \%$ the dual-bell is set into a nonstop flip-flop phenomenon that continues as long as the pressure fluctuations prevail inside the HASC. However for $f>1.5 \mathrm{~Hz}$, no full transition could be achieved in the present test arrangement although the dual-bell exhaust was observed to set into an oscillatory mode wherein the wall pressure in the entire nozzle extension fluctuated considerably at the same frequency. This behavior too is not favorable as it can delay the dual-bell transition process. Increasing the throttling up rate also does not seem to help much in achieving dual-bell transition within the scope of the present tests. However, more investigations need to be conducted to corroborate this. These test results suggest that the dualbell nozzle can present problems in real-flight operation as the launcher experiences the buffeting phase of flight. Therefore, a lot of care needs to be taken while designing the dual-bell nozzle for realflight situation so as to ensure a stable forward transition. The solution perhaps lies in increasing the dual-bell stability parameter $\mathrm{DB}_{\mathrm{st}}$ to a value greater than the magnitude of $P_{b}$ fluctuations (of $20 \%$ ) so as to avoid the NPR range that will set the dual-bell into a non-stop flip-flop mode during buffeting. This also shows that for a dual-bell nozzle, the magnitude of $P_{b}$ fluctuations based on the $\mathrm{DB}_{\mathrm{st}}$ parameter is an important parameter in addition to its frequency and cannot be ignored.

\section{Acknowledgements}

The results reported in this paper are from the work carried out by the first author as a revisiting Humboldt fellow in the Nozzle flow Group at Space Propulsion Institute, DLR Lampoldshausen, Germany from May 2010 to July 2010. The author would like to thank the Humboldt foundation for the same and his organization NAL for granting permission to go on deputation to carry out this work.

\section{References}

[1] Kasuka, K., Kumakawa, A., Niino, M., Konno, A. and Atsumi, M.,'Experimental Study on Extendible and Dual-Bell Nozzles Under High Altitude Conditions', AIAA 2002-3303.

[2] Foster, C. and Cowles, F., 'Experimental Study of Gas-Flow Separation in Overexpanded Exhaust Nozzles for Rocket Motors,' Jet Propulsion Laboratory, California Institute of Technology, Progress Report 4-103, 1949.

[3] Horn, M. and Fisher, S., “Dual-Bell Altitude Compensating Nozzle,” NASA CR-194719, 1994.

[4] G.V.R Rao, “Recent Developments in Rocket Nozzle Configurations”, ARS Journal, Nov. 1961, pp. 1488-1494.

[5] Wasko, R.A.,"Performance of Annular Plug and Expansion-Deflection Nozzles including External Flow Effects at Transonic Mach Numbers", NASA TN D-4462, April 1968. 
[6] Hagemann G., Immich H., Nguyen, T.V. and Dumnov, G.E.,"Advanced Rocket Nozzles”, Journal of Propulsion and Power, Vol. 14, No. 5, Sept-Oct. 1998, 629-634.

[7] Ruf, J. H. and McConnaughey, P. K.,' 'The Plume Physics Behind Aerospike Nozzle Altitude Compensation and Slipstream Effect", AIAA Paper 97-3217.

[8] Kalinin, E.M., Lapygin, V.I., Pushkin, R.M. and Aksenov, L.A.,“Gas Dynamics of SelfAdjustable Thruster with Zero-Length Central Plug', 1998, ESTEC, ESA SP-426

[9] Rao, GVR, “The E-D Nozzle’”, Astronautics, 28-29, 50-51, 1960

[10] Immich, H. and Caporicci, M., “FESTIP Technology Developments in Liquid Rocket Propulsion for Reusable Launch Vehicles,"' AIAA Paper 97-3311.

[11] Frey, M. and Hagemann, G.,'Critical Assesment of Dual-Bell Nozzles,'” Journal of Propulsion and Power, Vol. 15, No.1, Jan.-Feb. 1999.

[12] Dumonov, G., Ponomaryov, N.B. and Voinov, A.L.,"Dual-Bell Nozzles for Rocket Engines of Launch Vehicle Upper Stages and Orbital Transfer Vehicles,’’ AIAA Paper 97-3089.

[13] Hagemann, G., Immich, H. and Preuss, A, “Advanced Nozzle Concepts for Future Rocket Engine Applications", $4^{\text {th }}$ International Conference on Launcher Technology, 3-6 December 2002, Liege, Belgium.

[14] Nuerenberger-Genin, C. and Stark, R.,"Experimental Study on Flow Transition in Dual-Bell Nozzles", AIAA 2009-4855

[15] Chloe Nürnberger-Genin and Ralf Stark, "Flow transition in dual bell nozzles", Journal of Shock Waves (2009), Vol. 19, No. 3, pp.265-270.

[16] Nasuti, F., Onofri, M and Martelli, E.," Role of Wall Shape on the Transition in Axisymmetric Dual-Bell Nozzles", Journal of Propulsion and Power, Vol. 21, No. 2. pp. 243-250.

[17] Verma, S.B., Stark, R., Genin, C. and Haidn, O.,“Flow Separation Characteristics of a Dual-Bell Nozzle during its Transition Modes', Journal of Shock Waves, Vol. 20, Issue 3, June 2010, pp. 191203.

[18] Stark, R., Boehm, C., Haidn, O.J. and Zimmermann, H.,'Cold Flow Testing of Dual-Bell Nozzles in Altitude Simulation Chambers', EUCASS, July 2005, Moscow, Russia.

[19] Tomita, T., Takahashi, M., Sasaki, Masaki,' Control of Transition between Two Working Modes of a Dual-Bell Nozzle by Gas Injection', AIAA-2009-4952.

[20] Martelli, E., Scaramuzzino, F. and Nasuti, F.,'Secondary Gas Injection Effect on Dual-Bell Nozzle Flow Transition', Paper\# 996-1538-1-RV, 4th European Conference for aerospace Sciences (EUCASS), St. Petersburg, Russia, July 4-8, 2011.

[21] Verma, S.B., Stark R. and Oskar Haidn, “Gas Density Effects on Dual-Bell Transition Behavior", AIAA Journal of Propulsion and Power, vol. 28, No. 6 (2012), pp.1315-1323, doi: $10.2514 / 1.6033$

[22] Perigo, D., Schwane, R., Wong, H.,“A numerical comparison of the flow in conventional dual bell nozzles in the presence of an unsteady external pressure environment', AIAA-2003-4731 
[23] Ariane 5 User's Manual, Approved and Issued by Arianespace, Issue 3, Revision 0, March 2000.

[24] Verma, S.B., Stark, R. and Haidn, O.,' Relation Between Shock Unsteadiness and the Origin of Side-Loads in a Thrust Optimized Parabolic Rocket Nozzle", The Aerospace Science and Technology Journal, Vol. 10, Issue 6, September 2006.

[25] Verma, S.B. and Haidn, O.,"Flow Separation Characteristics of Overexpanded Nozzles", International Journal of Aerospace Innovations, Invited paper for a special issue on 'Overexpanded and Underexpanded Nozzles', Vol.2, No.4, December 2010, pp. 259-277.

[26] Verma, S.B., Stark, R. and Haidn, O., ' Reynolds Number Influence on Dual-Bell Transition Phenomena", AIAA Journal of Propulsion and Power, Vol.29, No.3, May-June 2013, pp.602-609. 


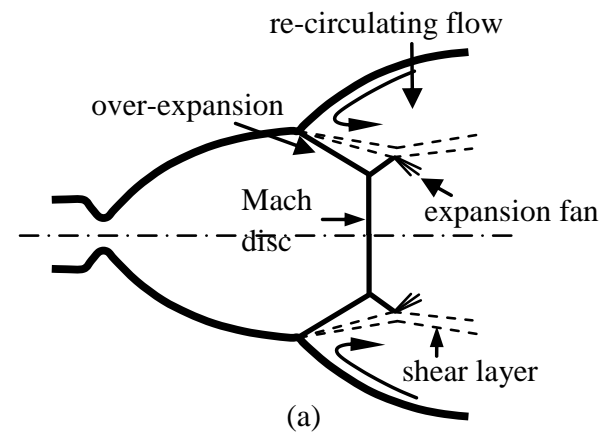

(a)

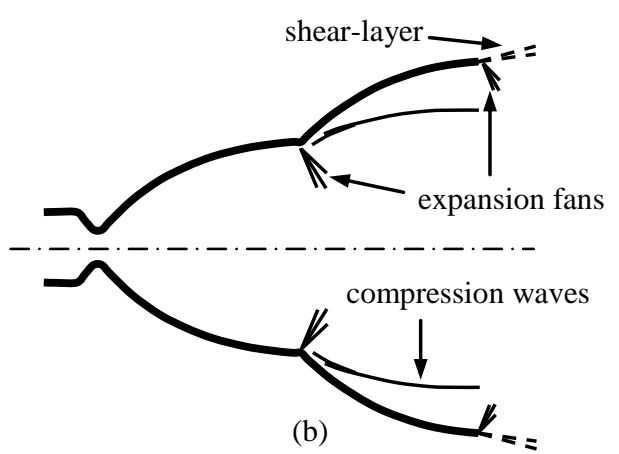

(b)

Figure 1: Schematic of the exhaust flow pattern from a dual-bell nozzle in (a) lowaltitude mode and, (b) high-altitude mode

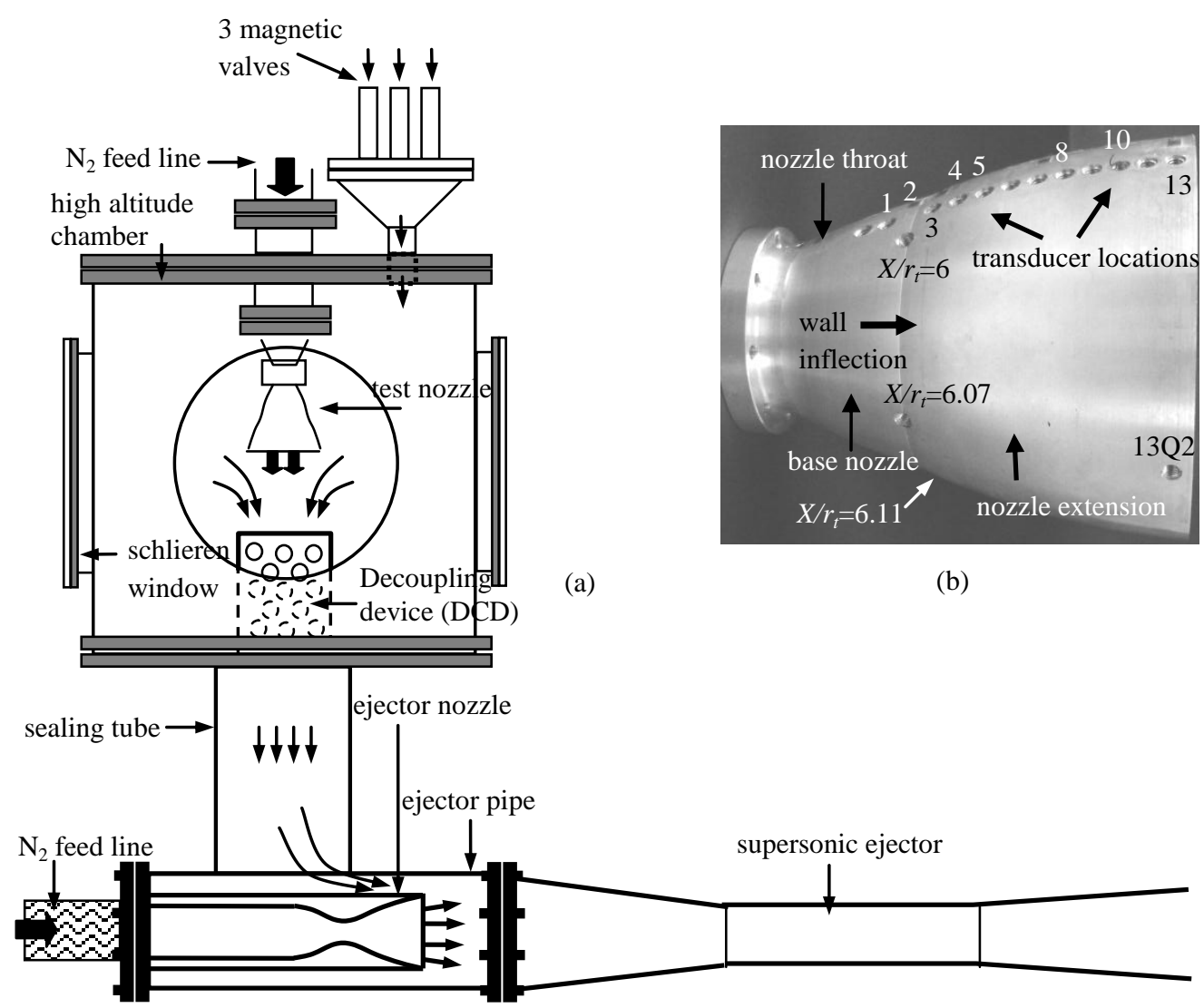

Figure 2: Dual-bell test nozzle showing (a) schematic of the high altitude simulation chamber (HASC) nozzle test-facility with nozzle operation in ejector mode and, (b) the pressure transducer locations

\begin{tabular}{|l|l|}
\hline Throat radius $\left(\mathrm{R}_{\mathrm{t}}\right)$ & $10 \mathrm{~mm}$ \\
Area-ratio $\left(\epsilon_{\mathrm{b}}\right)$ at inflection pt. (TIC) & 11.3 \\
Area-ratio $(\epsilon)$ at nozzle extension $(\mathrm{CP})$ & 27.1 \\
Wall angle at nozzle exit, $\alpha_{i}$ & $7.26^{\circ}$ \\
\hline Averaged transition NPR & 49 \\
Averaged re-transition NPR & 39 \\
\hline
\end{tabular}

Table 1: Dual-Bell Nozzle Details 


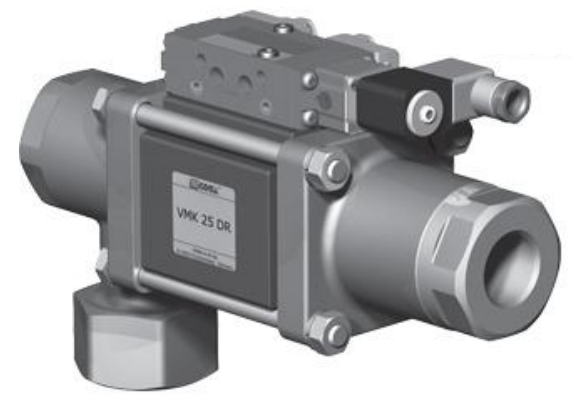

(a)

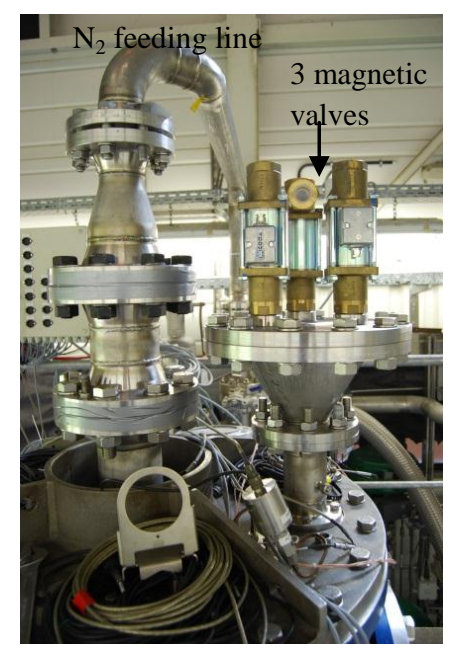

(b)

Figure 3: (a) Image showing the co-axial magnetic valve used to produce pressure fluctuations inside the HASC and, (b) 3 magnetic valves mounted on the HASC

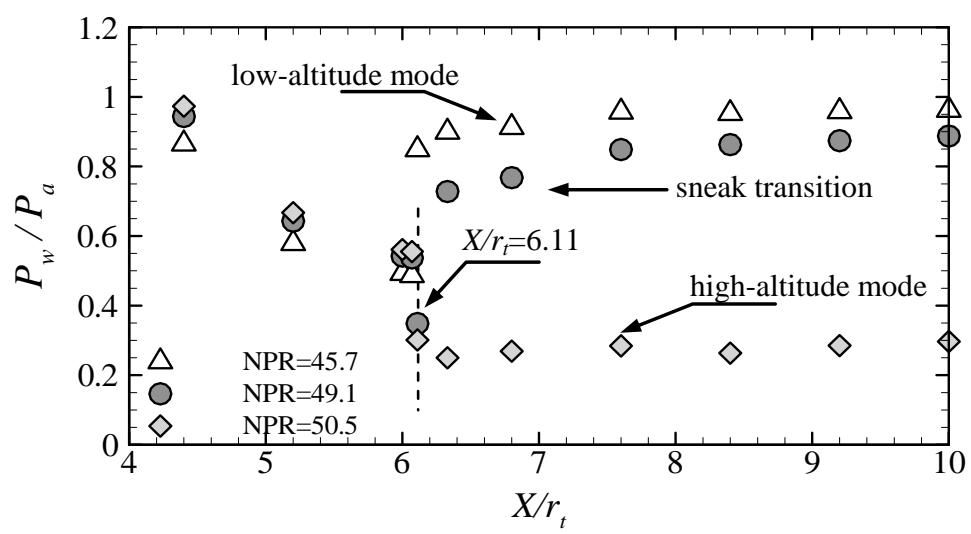

Figure 4: Streamwise distribution of mean wall pressure
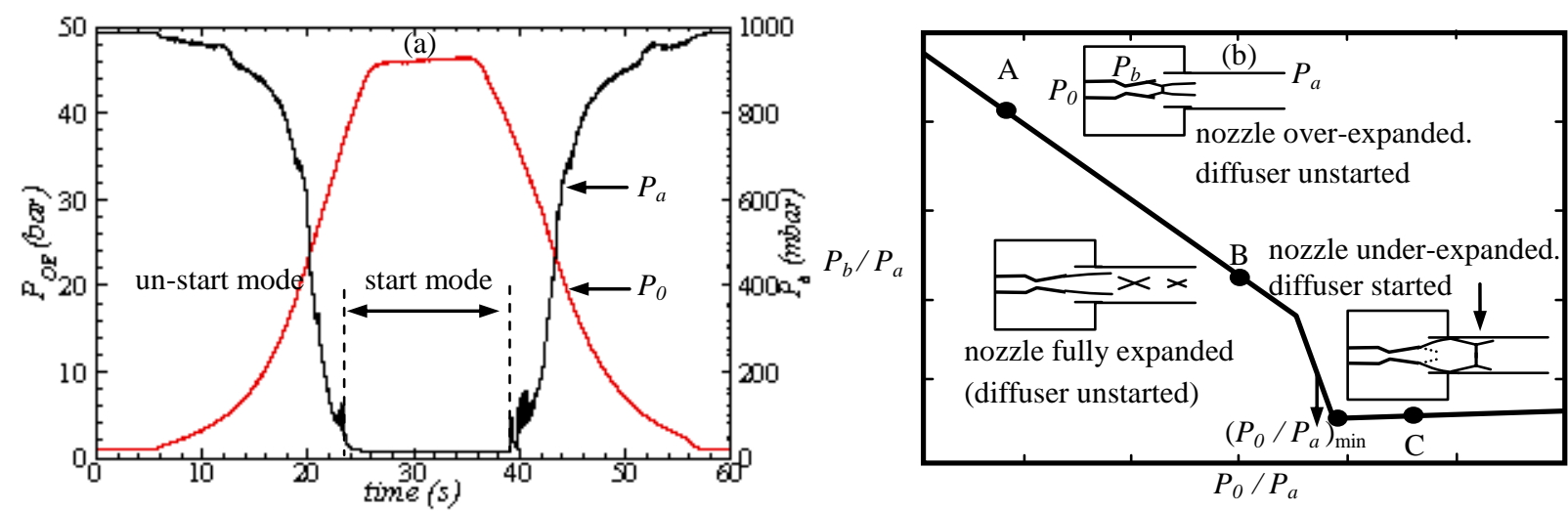

Figure 5: (a) Plot showing the variation in chamber pressure to changes in ejector nozzle pressure and (b) schematic of the diffuser operation inside a high altitude chamber 

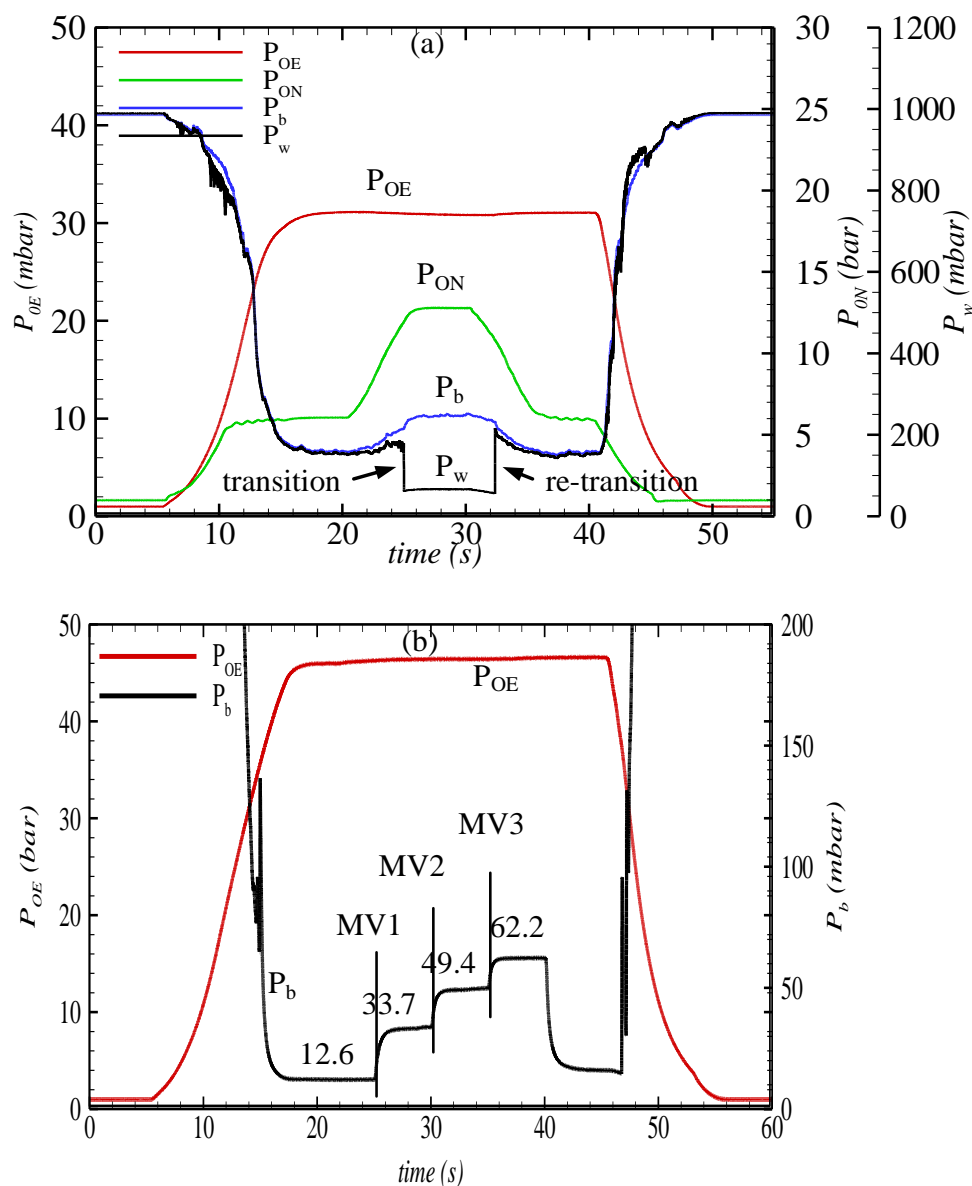

Figure 6: Plot showing (a) dual-bell operation without the use of magnetic valves (b) the variation in $P_{b}$ (mbar) when the magnetic valves are switched on one by one
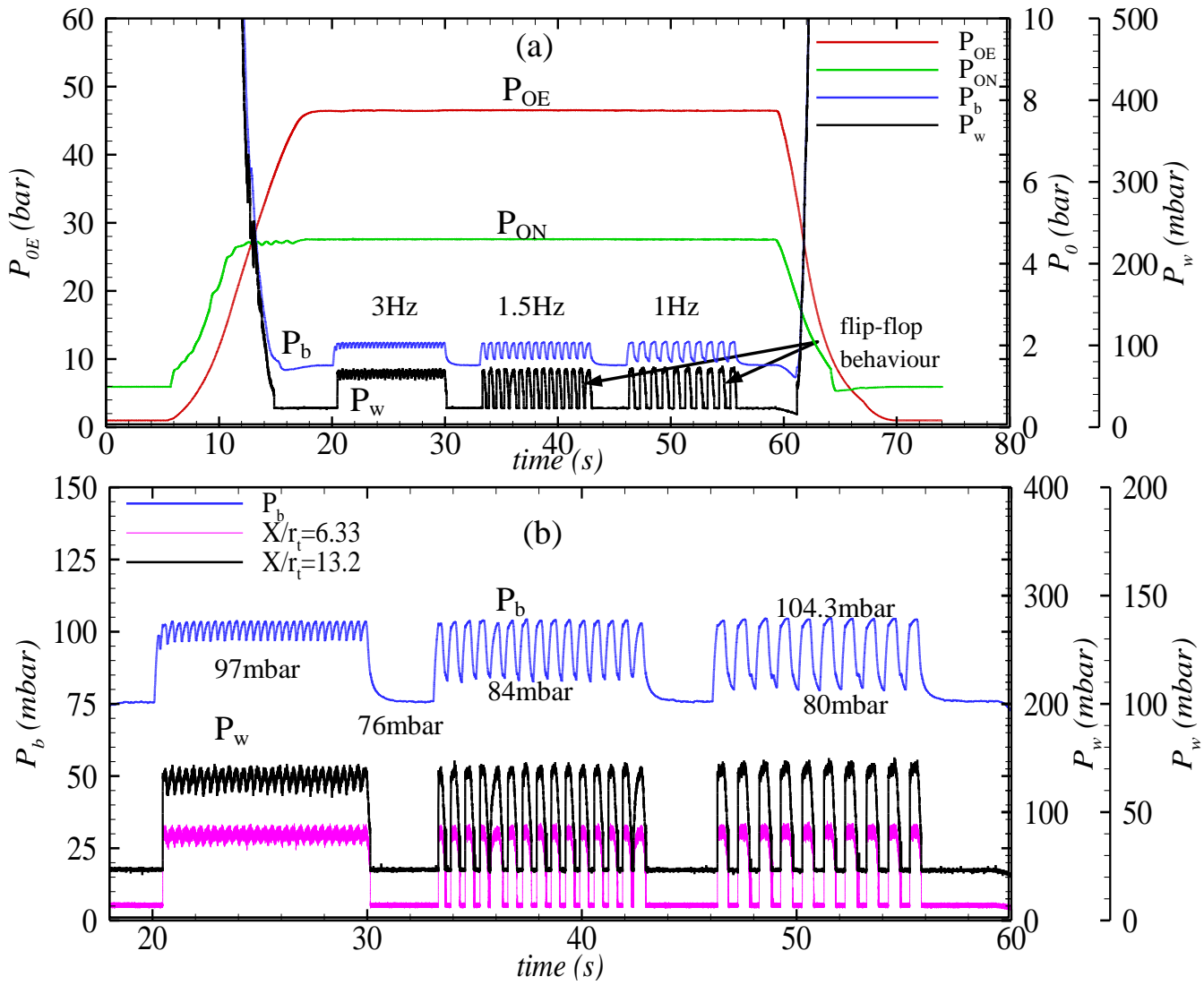


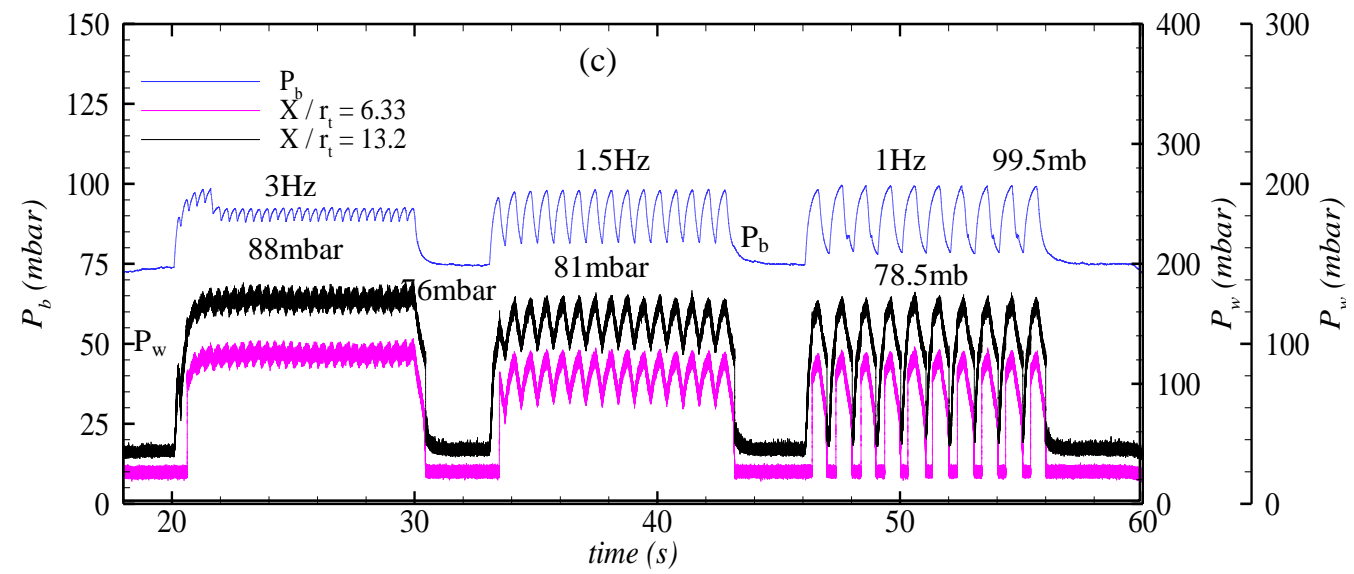

Figure 7: Plot showing (a) dual-bell operation with the use of 3 magnetic valves (b) variation in $P_{w}$ in nozzle extension in response to the fluctuations using $3 \mathrm{MVs}$ (c) variation in $P_{w}$ in nozzle extension in response to the fluctuations using $2 \mathrm{MVs}$; MV operation $\rightarrow$ off-on-off 

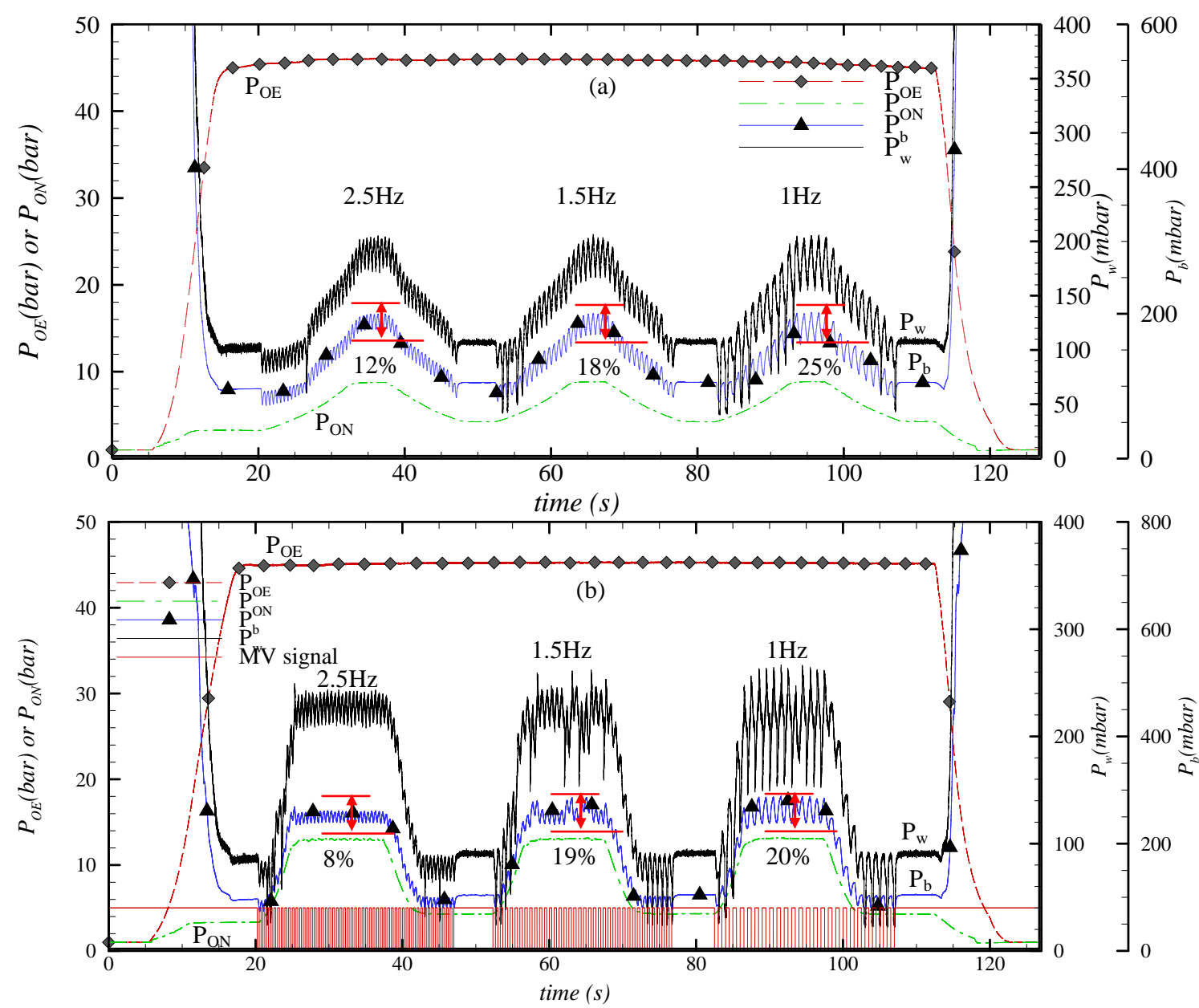

Figure 8: Plot showing the effect of throttling up and down in the presence of back pressure fluctuations (a) $\partial P_{O N} / \partial t=0.5 \mathrm{bar} / \mathrm{s}$ (b) $\partial P_{O N} / \partial t=2 \mathrm{bar} / \mathrm{s}$ 